\title{
Identifying Large Scale Features on the Surface of Contact Binaries with Sub-Arcsecond Techniques
}

\author{
P.G. Niarchos \\ University of Athens, Panepistimiopolis, GR 15784 Zografos, Athens, \\ Greece,pniarcho@cc.uoa.gr
}

\section{Pustylnik}

Tartu observatory, Tartu region 61602, Estonia, izold@aai.ee

\begin{abstract}
We summarize multifold evidence for the presence of a hot spot region in contact binary VW Cephei. We interpret this feature as a photospheric burn and the preferential site of flares in EUV and X rays. With its trigonometric parallax of 0.041 arcsec VW Cep is a promising target for a milliarcsecond resolution investigation of the nature of surface features both in optical region and in IR.
\end{abstract}

In our papers Pustylnik, Niarchos 1999, 2000 (respectively Paper I and II) we have summarized manifold observational data favouring the idea that a small size 'hot spot' residing on the surface of a more massive component close to the neck connecting two stars can naturally explain i) the pattern of the observed asymmetry in UBV light curves, ii) the phase variations of the colors $U-B, B-V$ and is in accord with the spectroscopic data on chromospheric activity signatures in this binary. Based on high quality observational data we found small, significant displacements of the brightness maxima in respect to the predicted moments of elongation. The displacement amounts to $0 .{ }^{\mathrm{d}} 005-$ $0 .{ }^{\mathrm{d}} 008$, i.e $0.03 P_{\text {orb }}$ (for more details see Paper II). For 9 observing nights a higher maximum (following the primary minimum) comes at a later epoch than it follows from the value of the orbital period, whereas the lower maximum (preceding the primary minimum) preferentially comes at an earlier epoch. This can be interpreted as an evidence for the asymmetric (in respect to the line of centres) brightness distribution over the hemisphere of a primary component facing its low mass companion. Displacement of the maxima positions from elongations along with the pronounced overall asymmetry of the light curves can be interpreted as an evidence of an additional energy input which effects the regular light variations caused by the tidal distortions. Whatever is the nature of mechanism responsible for the asymmetry, to cause the displacement in phase of maximum by $\Delta \phi$ an additional energy input is needed $L_{\text {add }} \geq C \frac{d l}{d \phi} \Delta \phi$, where the value $d l / d \phi$ can be estimated with the aid of the theoretical light curve and $C \simeq 1-10$. We have found $L_{\text {add }}=2 \cdot 10^{29} \mathrm{ergs} / \mathrm{s}$ and $L_{\text {add }}=2 \cdot 10^{30} \mathrm{ergs} / \mathrm{s}$ for $C=1$ and $C=10$ respectively. An average value of the difference between the heights of the adjacent maxima yields the estimate $L_{\text {add }}=3.5 \cdot 10^{30} \mathrm{ergs} / \mathrm{s}$. Assuming that mutual eclipses and tidal distortions fully determine the shape of the light curve for a standard limb darkening law we estimated the size and 
the temperature of the putative spot (small differences in temperature between the component stars and the gravity darkening effect were neglected). We have estimated the relative size $\delta_{\mathrm{sp}}$ of the hot spot and found that $\delta_{\mathrm{sp}} \simeq 0.02$ while its temperature is $T_{\mathrm{sp}}=7000 \mathrm{~K}$. For just quoted value of the size of hot spot and its temperature we have found that subsequent values for the amplitudes of colour changes between minimum and maximum $\Delta(U-B)=0.070(0.072), \Delta(B-V)=$ $0.038(0.040), \Delta(V-R)=0.040(0.030), \Delta(V-I)=0.049(0.044)$ (in brackets the observed values from Linnell 1982 are given). To verify how the hot spot with the above-given parameters can be helpful in interpreting the observed light curves of VW Cep we used commercially available computer package BINARY MAKER and modelled with its aid the light curves of VW Cep assuming the orbital and physical parameters from Hill 1989. We have found the best fit for the following values of the hot spot parameters: $R_{\mathrm{sp}}=7^{\circ} \pm 1^{\circ}, \delta T / T \simeq 1.35-1.4, L_{3}=0.02-$ 0.03 (the third light) $f=0.05, l=80^{\circ} \pm 2^{\circ}, \chi=357^{\circ} \pm 2^{\circ}$ ( $l$ being the latitude, $\chi$ the longitude of the centre of the hot spot, $f$ a fill-out parameter). The derived value of the temperature of the hot spot is practically coincident with the temperature needed to generate $M g I I \lambda 2795,2802 \dot{A}$ strong resonance doublet feature, whereas both the flux in this feature and the phase of its maximum are in good accord with the above given estimate of the luminosity of the hot spot and its location. We turn now to a short time intrinsic variability observed in VW Cep. Supposing that the hot spot region is the preferential site of the flare activity, assuming that the volume and emission measure remain constant during the flare we estimated the parameters of the flare in extreme UV (Rucinski 1998) and for the case of an optically thin plasma we calculated expected changes in colours $\Delta(U-B), \Delta(B-V), \Delta(V-R)$ between two measurements: one taken close to the peak of the flare and another one right after the flare. We have found $\delta[\Delta(U-B)]=-0.015, \delta[\Delta(B-V)]=0.007, \delta[\Delta(V-R)]=0.004$ (where $\delta$ stands for the variation of respective index between the two points). Thus, for realistic parameters of the flare the observed intrinsic colour changes of order $0^{\mathrm{m}} .01$ from one observational night to another (or within the same orbital cycle) can be easily reproduced.

Acknowledgements. We acknowledge with gratitude support of the current investigation by the Grant in the framework of the NATO Science Fellowship. One of us (I.P.) acknowledges also support by the Grant 2629 of Estonian Science Foundation.

\section{References}

Hill G. 1989, A\&A, 218, 141

Linnell A.P. 1982, ApJS, 50, 85

Pustylnik I. \& Niarchos P.G. 1999, Odessa Astron.Publ. 12, 238

Pustylnik I. E Niarchos P.G. 2000, A\&A, 361, 982

Rucinski S. 1998, AJ, 115, 303 